\title{
Observation of Zero-Point Quantum Fluctuations of a Single-Molecule Magnet through the Relaxation of its Nuclear Spin Bath
}

\author{
A. Morello, ${ }^{1,2, *}$ A. Millán, ${ }^{3}$ and L. J. de Jongh ${ }^{1}$ \\ ${ }^{1}$ Kamerlingh Onnes Laboratory, Leiden University, P.O. Box 9504, 2300RA Leiden, The Netherlands \\ ${ }^{2}$ Centre for Quantum Computation and Communication Technology, School of Electrical Engineering \\ and Telecommunications, UNSW Australia, Sydney, New South Wales 2052, Australia \\ ${ }^{3}$ Instituto de Ciencia de Materiales de Aragón, C.S.I.C. Universidad de Zaragoza, 50009 Zaragoza, Spain
}

(Received 6 November 2013; published 18 March 2014)

\begin{abstract}
A single-molecule magnet placed in a magnetic field perpendicular to its anisotropy axis can be truncated to an effective two-level system, with easily tunable energy splitting. The quantum coherence of the molecular spin is largely determined by the dynamics of the surrounding nuclear spin bath. Here we report the measurement of the nuclear spin-lattice relaxation rate $1 / T_{1 n}$ in a single crystal of the singlemolecule magnet $\mathrm{Mn}_{12}$-ac, at $T \approx 30 \mathrm{mK}$ in perpendicular fields $B_{\perp}$ up to $9 \mathrm{~T}$. The relaxation channel at $B \approx 0$ is dominated by incoherent quantum tunneling of the $\mathrm{Mn}_{12}$-ac spin $\mathbf{S}$, aided by the nuclear bath itself. However for $B_{\perp}>5 \mathrm{~T}$ we observe an increase of $1 / T_{1 n}$ by several orders of magnitude up to the highest field, despite the fact that the molecular spin is in its quantum mechanical ground state. This striking observation is a consequence of the zero-point quantum fluctuations of $\mathbf{S}$, which allow it to mediate the transfer of energy from the excited nuclear spin bath to the crystal lattice at much higher rates. Our experiment highlights the importance of quantum fluctuations in the interaction between an "effective twolevel system" and its surrounding spin bath.
\end{abstract}

PACS numbers: 75.50.Xx, 42.50.Lc, 75.45.+j, 76.60.Es

Quantum mechanical two-level systems (TLS) are the subject of vivid interest, motivated by their application in quantum information technology [1]. In this context, the model of a "central spin + spin bath" [2] is of widespread fundamental interest for a wide range of natural or artificial TLS [3]. Single-molecule magnets (SMMs) [4] constitute a prototypical example of mesoscopic quantum systems that, under suitable experimental conditions, can be treated as an effective TLS [5]. The "qubit levels" arise from the lowenergy truncation of the larger Hilbert space of a high-spin (typical $S \sim 10$ ) molecule. In the presence of uniaxial magnetocrystalline anisotropy, the resulting qubit energy splitting $\hbar \omega_{e}$ has a very strong dependence on the external magnetic field $B_{\perp}$ applied perpendicular to the anisotropy axis [6], $\hbar \omega_{e} \propto B_{\perp}^{2 S}$, making the splitting easily tunable. Being stoichiometric and crystalline compounds, SMMs are exquisitely suited for the fundamental study of decoherence in mesoscopic systems, because the Hamiltonian of the qubit and the environment (spin and phonon bath) is known in utmost detail. Indeed, a recent experiment found that the spin coherence of $\mathrm{Fe}_{8} \mathrm{SMMs}$ in large transverse field [7] is in excellent agreement with the most accurate theories [5].

Here we report a pulse-NMR study of the nuclear spin bath dynamics in a single crystal of the molecular magnet $\mathrm{Mn}_{12}$-ac SMMs at ultralow temperatures $(T \approx 30 \mathrm{mK})$ in strong perpendicular magnetic fields ( $B_{\perp}$ up to $\left.9 \mathrm{~T}\right)$. This allows us to explore the regime where the molecular spin (qubit) splitting $\hbar \omega_{e}$ becomes larger than all other relevant energy scales. We focus, however, on the bath rather than the qubit, and find strong evidence that zero-point quantum fluctuations (ZPFs) [8] of the molecular spin dominate the nuclear bath dynamics. We deduce this by measuring the transverse field dependence of the nuclear spin relaxation rate $1 / T_{1 n}\left(B_{\perp}\right)$. In a material such as $\mathrm{Mn}_{12}$-ac, nuclear spins do not have a channel for direct relaxation to the phonon bath - their relaxation must be mediated by electron spin fluctuations. When $\hbar \omega_{e} \gg k T$, thermal fluctuations of the electron spin become exponentially suppressed and one expects $1 / T_{1 n} \rightarrow 0$. Instead we find that $1 / T_{1 n}\left(B_{\perp}\right)$ increases by five orders of magnitude up to the highest field. Indeed, quantum mechanics predicts [9] that, since a fully polarized state with $\mathbf{S} \| \mathbf{B}_{\perp}$ is not an eigenstate of the spin Hamiltonian (unless $B_{\perp} \rightarrow \infty$ ), the central spin will exhibit quantum fluctuations down to $T=0$. This experiment thus represents an attractive physical implementation of the ideas discussed by Gavish et al. [10], who argued that although ZPFs cannot supply energy, they can indeed (even at $T \approx 0$ ) absorb energy when coupled to an activated system (here the nuclear spins, excited by NMR pulses), thereby deexciting it.

The properties of the $\mathrm{Mn}_{12}$-ac SMM are well known. We adopt the same electron spin Hamiltonian $\mathcal{H}_{S}$ and parameter values as in previous work $[11,12]$ :

$$
\begin{aligned}
\mathcal{H}_{S}= & D S_{z}^{2}+E\left(S_{x}^{2}-S_{y}^{2}\right)+B_{4} S_{z}^{4}+C\left(S_{+}^{4}+S_{-}^{4}\right) \\
& +\mu_{B} \mathbf{B}_{\perp} \cdot \hat{g} \cdot \mathbf{S} .
\end{aligned}
$$


We also add a term accounting for a dipolar field $B_{\mathrm{dd}}$ from neighboring molecules, of the form $\mathcal{H}_{\mathrm{dd}}=g_{z} \mu_{B} B_{\mathrm{dd}} S_{z} \approx$ $0.1 \mathrm{~K}$ [13]. The hyperfine interaction between nuclear and electron spins is $\mathcal{H}_{\text {hyp }}=-\mathbf{I} \cdot \hat{A} \cdot \mathbf{S}$, with $\hat{A}$ the hyperfine tensor. The $\mathrm{Mn}_{12}$-ac molecules in the crystal contain 4 $\mathrm{Mn}^{4+}$ ions (with ionic spin $s=3 / 2$ ), giving rise to the NMR resonance labeled $\mathrm{Mn} 1$, and $8 \mathrm{Mn}^{3+}$ ions $(s=2)$ [Fig. 1(a)], the latter occupying two inequivalent sites which give rise to different hyperfine interactions [14] and two separate resonance lines, $\mathrm{Mn} 2$ and $\mathrm{Mn} 3$. Intramolecular magnetic exchange interactions between the ionic spins yield a net effective spin $S=10$ for the molecular cluster at low $T$. Below $\sim 1 \mathrm{~K}$ only the lowest doublet of states $|\mathcal{G}\rangle,|\mathcal{E}\rangle$ is thermally occupied [11], justifying the truncation of the 'giant spin' to an effective qubit Hamiltonian [2]. When $B=0,|\mathcal{G}\rangle$ and $|\mathcal{E}\rangle$ are symmetric and antisymmetric quantum superpositions of the $m_{S}= \pm 10$ projections of the molecular spin along the $z$-axis. Their energy splitting arises from the (weak) offdiagonal terms in $\mathcal{H}_{S}$, which introduce a tunnel coupling $\Delta_{0}$
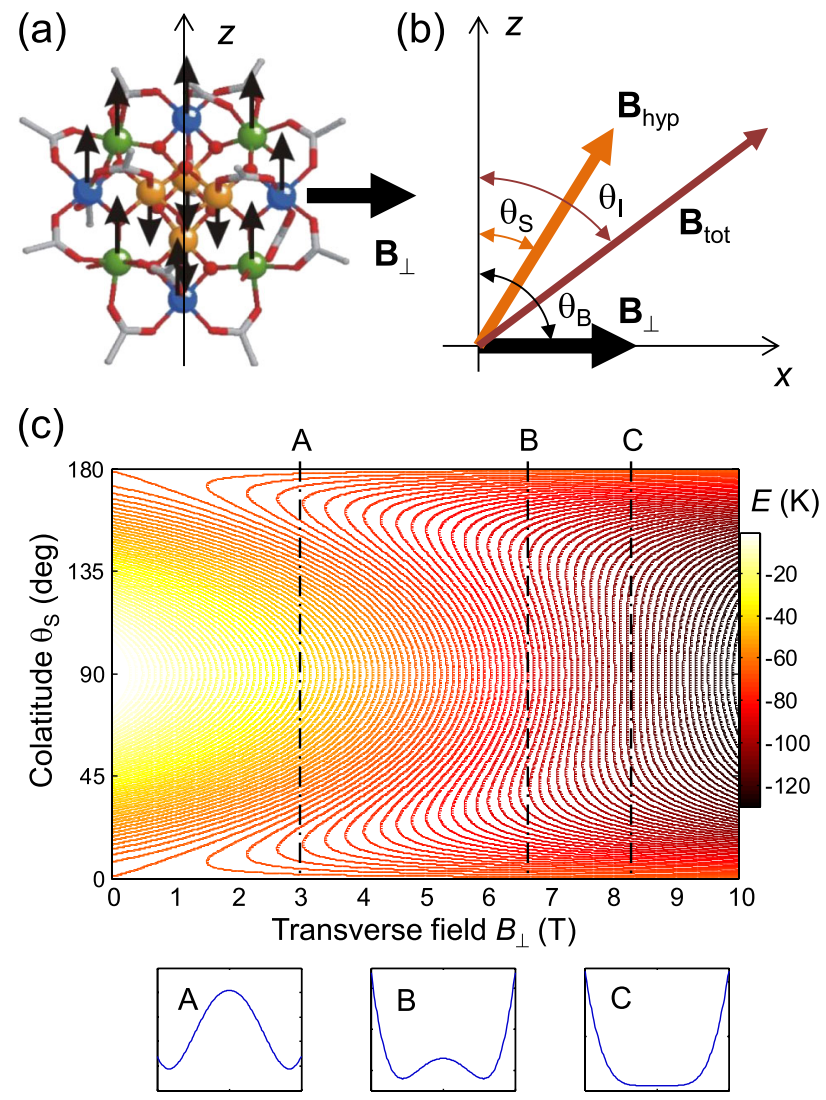

FIG. 1 (color online). (a) Structure of the $\mathrm{Mn}_{12}$-ac molecule. The 4 innermost Mn ions (spheres with down-arrows) give rise to the Mn1 NMR resonance line. (b) Orientation of the external $\left(\mathbf{B}_{\perp}\right)$, hyperfine $\left(\mathbf{B}_{\text {hyp }}\right)$ and total $\left(\mathbf{B}_{\text {tot }}\right)$ field on the Mn1 nuclei. (c) Evolution of the total energy $E$ (crystal field anisotropy + Zeeman term) of the $\mathrm{Mn}_{12}$-ac spin as a function of transverse field and angle $\theta_{S}$. Representative energy profiles at $B_{\perp}=3 \mathrm{~T}$ (A), $6.65 \mathrm{~T}(\mathrm{~B}), 8.25 \mathrm{~T}(\mathrm{C})$ are shown underneath. $\left(\sim 10^{-10} \mathrm{~K}\right.$ at $\left.B=0\right)$ between spin states at opposite sides of the classical spin anisotropy barrier [Fig. 1(c)]. In previous work [15] we showed that the nuclear spin bath can relax and thermalize via incoherent quantum tunneling of the central spin, which is itself driven by the internal dynamics of the nuclear bath [2], giving rise to nontrivial quantum relaxation effects [16]. It was also argued that the most of the dynamics at $B=0$ arises from a minority of fast-relaxing molecules (FRMs) $[15,17]$.

In the present study we apply a strong perpendicular field $B_{\perp} \| x$, which causes $|\mathcal{G}\rangle$ and $|\mathcal{E}\rangle$ to contain amplitudes from all $m_{S}=-10 \ldots+10$. They can still be written as a superposition of 'classical' states $\left|\mathcal{Z}_{ \pm}\right\rangle$corresponding to the two total energy minima [Fig. 1(c)], but the spin expectation values of $\left|\mathcal{Z}_{ \pm}\right\rangle$are now canted towards the $x$-axis [18], forming an angle $\theta_{S}=\sin ^{-1}\left(\left\langle S_{x}\right\rangle / S\right)$ with the $z$-axis [Fig. 1(b)]. The hyperfine interaction $\mathcal{H}_{\text {hyp }}$ is commonly written in terms of an effective field $\mathbf{B}_{\text {hyp }}=-\hat{A} \cdot \mathbf{S} / \hbar \gamma_{n}$. The total field at the nuclear site, $\mathbf{B}_{\text {tot }}=\mathbf{B}_{\perp}+\mathbf{B}_{\text {hyp }}$, defines the quantization axis for the nuclear spin and yields the nuclear Larmor frequency $\omega_{n}=2 \pi \nu_{n}=\gamma_{n}\left|\mathbf{B}_{\text {tot }}\right|$, with $\gamma_{n}$ the nuclear gyromagnetic ratio. We performed ${ }^{55} \mathrm{Mn}\left(I=\frac{5}{2}\right)$ and proton $\left(I=\frac{1}{2}\right)$ NMR experiments in the frequency range 220$330 \mathrm{MHz}$, in a cryogenic set-up described elsewhere $[11,19]$. The $\mathrm{Mn}_{12}$-ac single crystal was carefully oriented with its $z$-axis perpendicular to the magnetic field direction. Several attempts were made to fine-tune the crystal orientation, with no significant changes in the NMR measurement results.

Fig. 2 shows the evolution of the NMR frequencies with $B_{\perp}$. The Mn1 line is clearly split in two (1a and $1 \mathrm{~b}$ in Fig. 2), as also observed in other $\mathrm{Mn}_{12}$-ac single crystal studies in zero field [11,20]. Its field dependence is explained by a progressive canting of the total spin by $B_{\perp}$, and assuming $S=10$ at all fields [18]. The strong

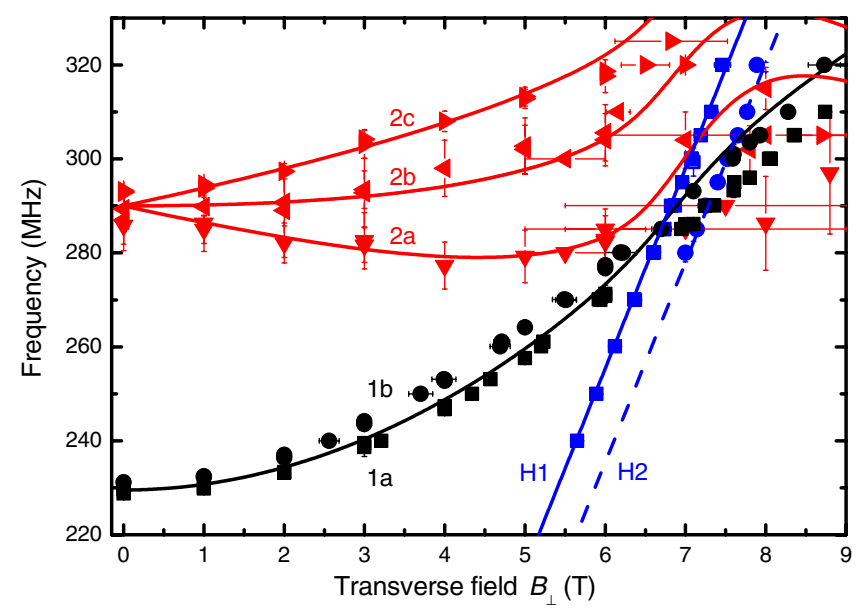

FIG. 2 (color online). Field dependence of the Mn1, Mn2 and ${ }^{1} \mathrm{H}$ NMR resonances (symbols) and FWHM (error bars, horizontal or vertical, from swept-field and -frequency runs, respectively). Lines are simulations discussed in Supplemental Material [12]. 
splitting of the Mn2 line was not observed in a previous experiment on aligned powder [18], but is readily explained on basis of the presence of the anisotropic dipolar hyperfine field [12,14]. The Mn3 line falls mostly outside our measurement window and will not be discussed. Two proton lines enter the measurement window when $5 \leq B_{\perp} \leq 8 \mathrm{~T}$. The main one (H1), responsible for $\approx 90 \%$ of the estimated total proton intensity, is observed at the unshifted frequency $\gamma B_{\perp} / 2 \pi$, implying a (dipolar) hyperfine field $B_{\text {dip }} \approx 0.1 \mathrm{~T}$ or less. The second line (H2) is shifted by $-19 \mathrm{MHz}$, corresponding to a $B_{\text {dip }} \approx 0.5 \mathrm{~T}$, in agreement with earlier deuteron NMR data [21], taking into account the difference in nuclear moments. The calculated ${ }^{55} \mathrm{Mn}$ spectra, shown as solid lines in Fig. 2, agree well with the data when assuming $\mathbf{B}_{\perp}$ makes an angle $\approx 90^{\circ} \pm 2^{\circ}$ with the $z$-axis.

The longitudinal nuclear spin relaxation (NSR) rate, $1 / T_{1 n}$, was measured with inversion recovery sequences, $\pi-t-\pi / 2-\tau-\pi$ [12]. A compilation of the NSR rates as a function of $B_{\perp}$ for $\mathrm{Mn} 1, \mathrm{Mn} 2$ and $\mathrm{H} 1$ is given in Fig. 3. We shall concentrate on the Mn1 line, which we were able to follow from $B_{\perp}=0$ to $9 \mathrm{~T}$, but all lines behave similarly in the field range where they could be observed. For moderate $B_{\perp}, 1 / T_{1 n}$ initially decreases, consistently with the interpretation that the $B \approx 0$ relaxation processes are driven by fast-relaxing molecules [15], whose easy axis is misaligned with that of the crystal. However, above $\sim 5 \mathrm{~T}$ a spectacular increase of a factor $10^{4}-10^{5}$ is seen in the $1 / T_{1 n}$ of all lines, all the way to $9 \mathrm{~T}$. To appreciate the significance of this observation, let us recall the standard expression of the NSR rate for nuclear spins coupled to a paramagnetic electron spin [22],

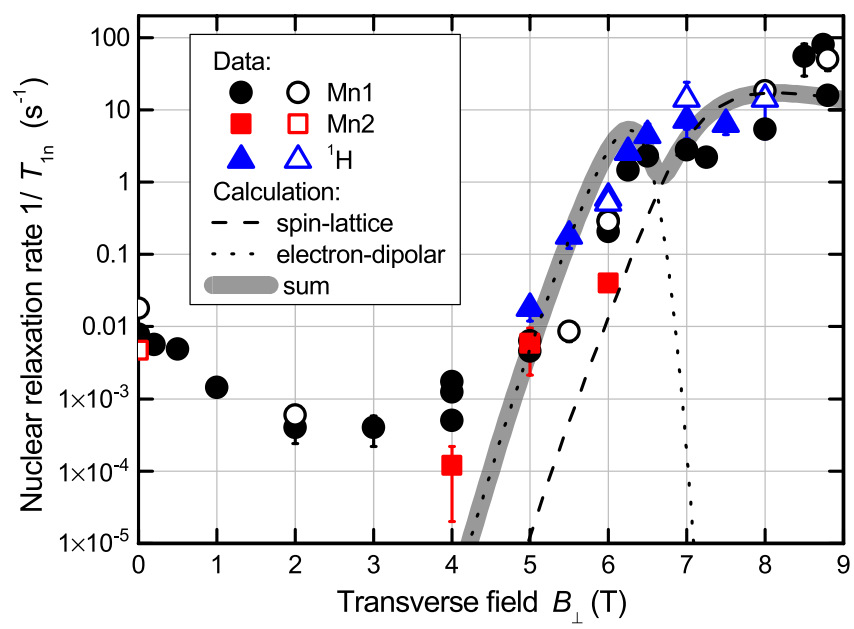

FIG. 3 (color online). (a) Nuclear spin relaxation rates $1 / T_{1 n}$ versus $B_{\perp}$ for $\mathrm{Mn} 1, \mathrm{Mn} 2$, and $\mathrm{H} 1$. Open symbols are data taken at a slightly $\left(\sim 2^{\circ}\right)$ different crystal orientation. Dashed line: calculated $1 / T_{1 n}$ arising from direct electron spin-lattice relaxation. Dotted line: calculated $1 / T_{1}{ }^{\star}$ from two-step relaxation through the electron dipolar reservoir. Thick gray line: sum of both contributions.

$$
\frac{1}{T_{1 n}} \approx \frac{1}{T_{1 e}}\left(\frac{A_{\perp}}{\omega_{n}}\right)^{2}\left(1-P_{e}^{2}\right),
$$

where $A_{\perp}$ is the off-diagonal part of the hyperfine coupling, $P_{e}=\tanh \left(\hbar \omega_{e} / 2 k T\right)$ is the electron spin polarization, and $1 / T_{1 e}$ is the electron spin-lattice relaxation rate [5]. For $\mathrm{Mn}_{12}$-ac the latter is given by

$\frac{1}{T_{1 e}} \simeq \frac{4 D^{2}\left|\left\langle\mathcal{E}\left|S_{x} S_{z}+S_{z} S_{x}\right| \mathcal{G}\right\rangle\right|^{2}\left(\hbar \omega_{e}\right)^{3}}{3 \pi \rho c_{s}^{5} \hbar^{4}} \operatorname{coth}\left(\frac{\hbar \omega_{e}}{2 k_{\mathrm{B}} T}\right)$,

where $\rho=1.83 \times 10^{3} \mathrm{~kg} / \mathrm{m}^{3}$ is the density and $c_{s} \simeq 1.5 \times$ $10^{3} \mathrm{~m} / \mathrm{s}$ is the sound velocity. In Fig. 4(b) we plot the calculated values of $1 / T_{1 e}$ and $\left(1-P_{e}^{2}\right)$ as a function of $B_{\perp}$. Although $1 / T_{1 e}$ does increase with field, $\left(1-P_{e}^{\frac{1}{2}}\right)$ decreases much more dramatically for $B_{\perp}>6.5 \mathrm{~T}$. Naively applying Eq. (2) to our system would lead to the (incorrect) prediction of astronomically long nuclear spin relaxation times for $B_{\perp}>7 \mathrm{~T}$. Therefore we need to reconsider the problem of nuclear spin-lattice relaxation, taking proper account of the complex nature of the $\mathrm{Mn}_{12}$-ac electronic spin $\mathbf{S}$, and recognizing that the factor $\left(1-P_{e}^{2}\right)$ in
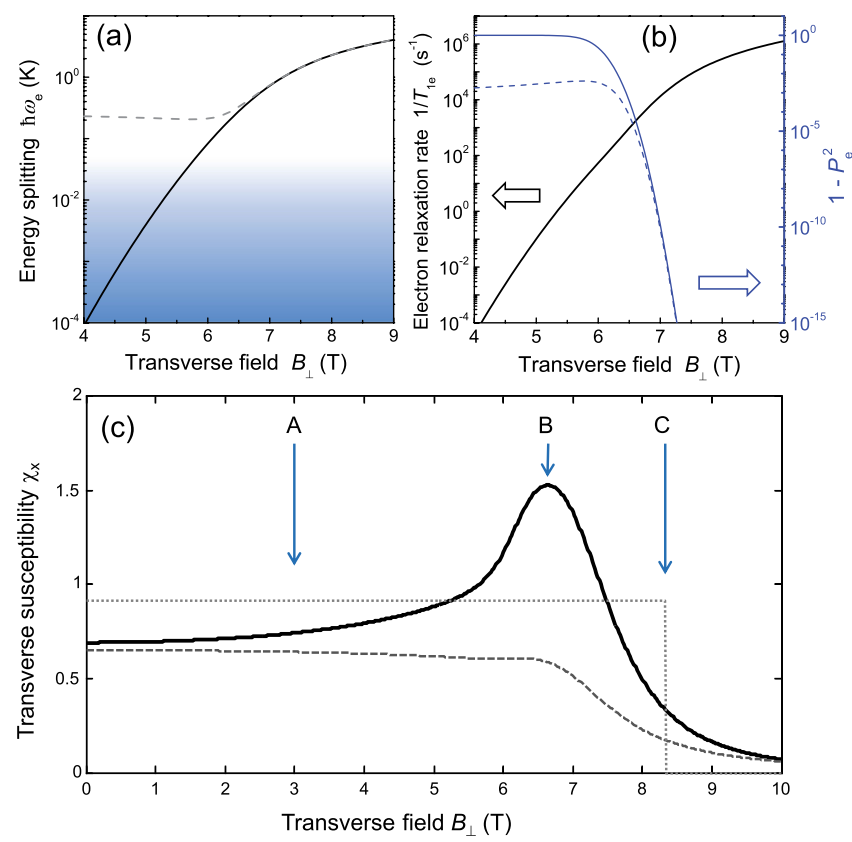

FIG. 4 (color online). (a) Energy splitting $\hbar \omega_{e}$ of the lowest electron spin state of the $\mathrm{Mn}_{12}$-ac molecule. Solid line: tunnel splitting $2 \Delta_{0}$. Dashed line: splitting including a longitudinal dipolar bias field, $\hbar \omega_{e}=\sqrt{\left(g \mu_{B} B_{\mathrm{dd}}\right)^{2}+2 \Delta_{0}^{2}}$. For $B_{\perp}>6.5 \mathrm{~T}$, $2 \Delta_{0}$ exceeds both $g \mu_{B} B_{\mathrm{dd}}$ and $k_{B} T(T \approx 30 \mathrm{mK}$, shaded area). (b) Calculated electron spin relaxation rate $1 / T_{1 e}$ (left scale) and $\left(1-P_{e}^{2}\right)$ (right scale), where $P_{e}$ is the electron spin polarization. The dotted line includes the contribution of $\mathcal{H}_{\mathrm{dd}}$. (c) $\mathrm{Mn}_{12}$-ac spin susceptibilities. Solid line: full quantum mechanical calculation of $\chi_{x}$. Dashed: spin fluctuations $\left\langle S_{x}^{2}\right\rangle-\left\langle S_{x}\right\rangle^{2}$. Dotted: classical mean-field value. Arrows show the points corresponding to the anisotropy potentials A, B, C in Fig. 1(c) as indicated. 
Eq. (2) represents in fact the differential electronic susceptibility $\chi_{\alpha}=g \mu_{B} \partial\left\langle S_{\alpha}\right\rangle / \partial B_{\alpha}$, with $\alpha$ the direction of the applied field.

The nuclear magnetization is relaxed via random fluctuations $\delta B_{\text {hyp }}(t)=-\left(A_{\perp} / \hbar \gamma_{n}\right) \delta S(t)$ in the hyperfine fields, associated with the electron spin fluctuations $\delta S(t)$. The NSR rate is obtained in perturbation theory as the Fourier transform of the electron spin correlation functions, evaluated at the nuclear Larmor frequency $\omega_{n}$,

$$
\begin{gathered}
\frac{1}{T_{1 n}}=\frac{1}{2}\left(\frac{A_{\perp}}{\hbar}\right)^{2} \bar{F}_{\alpha}\left(\omega_{n}\right), \\
\bar{F}_{\alpha}(\omega)=\int\left\langle\left\{\delta S_{\alpha}(0) \delta S_{\alpha}(t)\right\}\right\rangle e^{-i \omega t} d t(\alpha=x, y, z) .
\end{gathered}
$$

Here \langle\rangle denotes the thermal statistical average and \{\} the symmetrized spin operator product; $\bar{F}(\omega)=(1 / 2)[F(\omega)+$ $F(-\omega)]$ is the symmetrized quantum spectral density $[8,10,23]$ and positive (negative) frequencies correspond to absorption (emission) of energy [24]. Detailed balance requires $F(\omega)=e^{\hbar \omega / k_{B} T} F(-\omega)$, and, thus $\bar{F}(\omega)=$ $(1 / 2) \operatorname{coth}\left(\hbar \omega / 2 k_{B} T\right)[F(\omega)-F(-\omega)]$, leading to the low- $T$ quantum version of the fluctuation-dissipation theorem $[8,23]$,

$$
\bar{F}_{\alpha}(\omega)=\frac{\hbar}{g^{2} \mu_{B}^{2}} \operatorname{coth}\left(\frac{\hbar \omega}{2 k_{B} T}\right) \chi_{\alpha}^{\prime \prime}(\omega),
$$

relating spin fluctuations to the imaginary component $\chi^{\prime \prime}$ of the complex magnetic susceptibility. Using the Debye frequency distribution and focusing on the transverse susceptibility $\chi_{x}$, we write $\chi_{x}^{\prime \prime}(\omega)=\chi_{x}(0) \omega T_{1 e} /$ $\left(1+\omega^{2} T_{1 e}^{2}\right)$ and arrive at the final expression for the field-dependent NSR,

$$
\frac{1}{T_{1 n}\left(B_{\perp}\right)}=\frac{\hbar}{2}\left(\frac{A_{\perp}}{g \mu_{B}}\right)^{2} \operatorname{coth}\left(\frac{\hbar \omega_{n}}{2 k_{B} T}\right) \chi_{x} \frac{\omega_{n} T_{1 e}}{1+\omega_{n}^{2} T_{1 e}^{2}},
$$

where $A_{\perp}, \omega_{n}, T_{1 e}$ and $\chi_{x}$ are all functions of $B_{\perp}$. From the $\mathrm{Mn}_{12}$-ac Hamiltonian (1), we calculate numerically $\chi_{x}\left(B_{x}\right)=g \mu_{B} \partial\left\langle S_{x}\right\rangle / \partial B_{x}$ [solid line in Fig. 4(c)] and use it in Eq. (7) to obtain the NSR shown as the dashed line in Fig. 3. The calculation correctly predicts the strong increase in $1 / T_{1 n}$ up to the highest $B_{\perp}$.

The situation most often found in the literature has $\alpha=z$, in which case the electron Zeeman term $g \mu_{B} B_{z} S_{z}$ commutes with the strong uniaxial anisotropy term $D S_{z}^{2}$, $\hbar \omega_{e}$ is just the electron Zeeman splitting, and the susceptibility $\chi_{z}$ becomes the factor $\left(1-P_{e}^{2}\right)$ in Eq. (2), which vanishes in high field as $\exp \left(-\hbar \omega_{e} / k_{B} T\right)$. Our experiment is unique in that we have $\alpha=x$ and thus $g \mu_{B} B_{x} S_{x}$ does not commute with $D S_{z}^{2}$. The electronic splitting $\hbar \omega_{e}$ is now the quantum tunneling splitting, and the transverse susceptibility $\chi_{x}$ [Fig. 4(c)] remains finite up to $B_{\perp} \rightarrow \infty$ even at $T=0$.
To further clarify the special role of ZPFs in our system, we recall that the susceptibility $\chi_{x}(0)$ can also be obtained from the static isothermal magnetization (per molecule) $\left\langle M_{x}\right\rangle=\sum_{i}\left(-\partial E_{i} / \partial B_{x}\right) e^{-\beta E_{i}} / \sum_{i} e^{-\beta E_{i}} \quad\left(\beta=1 / k_{B} T\right.$, and $E_{i}$ are the electronic energy levels) as

$$
\chi_{x}(0)=\beta\left\langle M_{x}^{2}\right\rangle-\beta\left\langle M_{x}\right\rangle^{2}+\left\langle-2 \partial E_{i} / \partial B_{x}^{2}\right\rangle .
$$

The term $\left\langle-2 \partial E_{i} / \partial B_{x}^{2}\right\rangle$ is the Van Vleck susceptibility, and describes a change in magnetization which does not originate from a change in thermal population of the eigenstates. Although often neglected, it plays a crucial role in our case since it persists even to $T=0$, and is responsible for the pronounced increase in $\chi_{x}$ around $B_{\perp} \approx 6.65 \mathrm{~T}$, where the most drastic restructuring of the energy spectrum takes place [25]. The susceptibility peak signals the onset of strong ZPF of the $\mathrm{Mn}_{12}$-ac in the shallow double-well potential [9]. The classical mean-field susceptibility $\chi_{x}\left(B_{\perp}\right)=\left(g \mu_{B}\right)^{2} / 2 D$ [Fig. 4(c), dotted line] goes to zero for $B_{\perp}>2 D S / g \mu_{B}\left(=8.32 \mathrm{~T}\right.$ in $\mathrm{Mn}_{12}$-ac $)$ and is clearly insufficient to explain the finite NSR up to $9 \mathrm{~T}$. Neglecting the Van Vleck term from (8) is equivalent to considering only fluctuations of the form $\left\langle S_{x}^{2}\right\rangle-\left\langle S_{x}\right\rangle^{2}$ [Fig. 4(c), dashed line].

An additional relaxation mechanism can arise due to the dipole-dipole coupling between molecular spins, as recently discussed in the case of the isotropic $\mathrm{Mn}_{6}$ molecule [26]. In this process, nuclear spin energy is first shared very rapidly with the electron-dipolar (ED) reservoir, then the combined ED + nuclear-Zeeman (NZ) system relaxes to the lattice at the rate

$$
\frac{1}{T_{1}^{\star}}=\frac{1}{T_{1 e}} \frac{C_{E D}}{C_{N Z}+C_{E D}},
$$

where $C_{\mathrm{ED}}$ and $C_{\mathrm{NZ}}$ are the (field-dependent) specific heats of the ED and NZ reservoirs. We calculate $C_{\mathrm{NZ}}$ for the ${ }^{55} \mathrm{Mn}$ as in Eq. 3 of Ref. [26], and take for $C_{\mathrm{ED}}$ the Schottky curve for a two-level system. The resulting $1 / T_{1}^{\star}$ is plotted as dotted line in Fig. 3. It drops markedly for $B_{\perp}>6.5 \mathrm{~T}$ due to the exponential decrease of $C_{\mathrm{ED}} / R \approx$ $\left(\hbar \omega_{e} / k_{B} T\right)^{2} e^{-\hbar \omega_{e} / k_{B} T}$ for $\hbar \omega_{e} \gg k_{B} T$, but it gives a significant contribution at lower fields. Taken together (thick gray line in Fig. 3), the calculated values of the NSR from Eqs. (7) and (9) are in remarkable qualitative and even quantitative agreement with the Mn1 data for $B_{\perp} \gtrsim 5 \mathrm{~T}$. We stress that no free fitting parameters were used at any point.

The-perhaps surprising — similarity between the Mn1, $\mathrm{Mn} 2$, and $\mathrm{H} 1$ relaxation rates (in the field range where data are available on all of them) can be related to the similar order of magnitude of the nondiagonal term $\left(A_{\perp} / \hbar \omega_{n}\right)^{2}$ in Eq. (7). The value of $A_{\perp}$ depends on the angle between $B_{\text {hyp }}$ and $B_{\text {tot }}$ [18], $A_{\perp}=A\left|\sin \left(\theta_{I}-\theta_{S}\right)\right|$ [see Fig. 1(b)], as well as on the presence of a nondiagonal component in the 
hyperfine coupling tensor, more pronounced in $\mathrm{Mn} 2$ and $\mathrm{H} 1$.

In conclusion, we have shown that the relaxation of the nuclear spin bath in $\mathrm{Mn}_{12}$-ac, at very low temperature, accelerates spectacularly when a large transverse field greatly enhances the zero-point quantum fluctuations of the molecular spin, whereas the electronic susceptibility remains finite up to the highest applied field. The associated zero-point quantum fluctuations of the molecular spin provide a dynamics persisting even down to $T=0$ and thus a very effective channel for de-excitation of the nuclear spin bath, even when the $\mathrm{Mn}_{12}$-ac spin can be considered as an effective two-level system in its ground state with a large gap to the first excited state. In addition to providing an appealing test bed for recent theories [10], our experiment highlights a profound difference between "true" $S=1 / 2$ spin qubits $[27,28]$ and effective TLSs that arise from the low-energy truncation of more complex systems.

This work has been part of the research program of the "Stichting FOM." We thank D. Bono for performing part of the experiments described. We acknowledge illuminating discussions with Y. Imry and R.S. Schoelkopf.

* Corresponding author. a.morello@unsw.edu.au

[1] T. D. Ladd, F. Jelezko, R. Laflamme, Y. Nakamura, C. Monroe, and J. L. O'Brien, Nature (London) 464, 45 (2010).

[2] N. V. Prokof'ev and P. C. E. Stamp, Rep. Prog. Phys. 63, 669 (2000).

[3] I. Buluta, S. Ashhab, and F. Nori, Rep. Prog. Phys. 74, 104401 (2011).

[4] D. Gatteschi and R. Sessoli, Angew. Chem., Int. Ed. 42, 268 (2003).

[5] A. Morello, P. C. E. Stamp, and I. S. Tupitsyn, Phys. Rev. Lett. 97, 207206 (2006).

[6] I. Ya. Korenblit and E. F. Shender, Sov. Phys. JETP 48, 937 (1978).

[7] S. Takahashi, I. S. Tupitsyn, J. van Tol, C. C. Beedle, D. N. Hendrickson, and P. C. E. Stamp, Nature (London) 476, 76 (2011).

[8] A. A. Clerk, M. H. Devoret, S. M. Girvin, F. Marquardt, and R. J. Schoelkopf, Rev. Mod. Phys. 82, 1155 (2010).

[9] A. K. Zvezdin, V. V. Dobrovitski, B. N. Harmon, and M. I. Katsnelson, Phys. Rev. B 58, R14733 (1998).
[10] U. Gavish, Y. Levinson and Y. Imry, Phys. Rev. B 62, R10637 (2000).

[11] A. Morello and L. J. de Jongh, Phys. Rev. B 76, 184425 (2007).

[12] See Supplemental Material at http://link.aps.org/ supplemental/10.1103/PhysRevLett.112.117202 for (1) experimental methods and analysis of nuclear spin relaxation measurements, (2) $\mathrm{Mn}_{12}$-ac spin Hamiltonian parameters, (3) measurement and calculation of NMR spectra.

[13] I. S. Tupitsyn, arXiv:cond-mat/0408220.

[14] T. Kubo, T. Goto, T. Koshiba, K. Takeda, and K. Awaga, Phys. Rev. B 65, 224425 (2002).

[15] A. Morello, O. N. Bakharev, H. B. Brom, R. Sessoli, and L. J. de Jongh, Phys. Rev. Lett. 93, 197202 (2004).

[16] W. Wernsdorfer, T. Ohm, C. Sangregorio, R. Sessoli, D. Mailly, and C. Paulsen, Phys. Rev. Lett. 82, 3903 (1999).

[17] W. Wernsdordfer, R. Sessoli and D. Gatteschi, Europhys. Lett. 47, 254 (1999).

[18] Y. Furukawa, K. Watanabe, K. Kumagai, F. Borsa, T. Sasaki, N. Kobayashi, and D. Gatteschi, Phys. Rev. B 67, 064426 (2003).

[19] A. Morello, W. G. J. Angenent, G. Frossati, and L. J. de Jongh, Rev. Sci. Instrum. 76, 023902 (2005).

[20] A. G. Harter, N. E. Chakov, B. Roberts, R. Achey, A. Reyes, P. Kuhns, G. Christou, and N. S. Dalal, Inorg. Chem. 44, 2122 (2005).

[21] J. Dolinšek, D. Arčon, R. Blinc, P. Vonlanthen, J. L. Gavilano, H. R. Ott, R. M. Achey, and N. S. Dalal, Europhys. Lett. 42, 691 (1998).

[22] See e.g., A. Abragam, and M. Goldman, Rep. Prog. Phys. 41, 395 (1978).

[23] R. Kubo, Rep. Prog. Phys. 29, 255 (1966).

[24] R. Aguado and L. P. Kouwenhoven, Phys. Rev. Lett. 84, 1986 (2000).

[25] O. B. Zaslavskiı̌, V. V. Ul'yanov, and V. M. Tsukernik, Sov. J. Low Temp. Phys. 9, 259 (1983).

[26] A. Morello, F. L. Mettes, O. N. Bakharev, H. B. Brom, L. J. de Jongh, F. Luis, J. F. Fernández, and G. Aromí, Phys. Rev. B 73, 134406 (2006).

[27] F. H. L. Koppens, C. Buizert, K. J. Tielrooij, I. T. Vink, K. C. Nowack, T. Meunier, L.P. Kouwenhoven, and L. M. K. Vandersypen, Nature (London) 442, 766 (2006).

[28] J. J Pla, K. Y. Tan, J. P. Dehollain, W. H. Lim, J. J. L. Morton, D. N. Jamieson, A. S. Dzurak, and A. Morello, Nature (London) 489, 541 (2012). 